\title{
Publisher Correction to: Marked reduction in the number of white globe appearance lesions in the noncancerous stomach after exchanging vonoprazan for esomeprazole treatment: a follow-up case report
}

\author{
Wataru Miwa ${ }^{1} \cdot$ Takashi Hiratsuka $^{1} \cdot$ Ken Sato $^{2} \cdot$ Takashi Fujino $^{3} \cdot$ Yo Kato $^{4}$
}

Published online: 18 June 2021

๑) Japanese Society of Gastroenterology 2021

\section{Publisher Correction to: Clinical Journal of Gastroenterology https://doi.org/10. 1007/s12328-021-01437-z}

We regret that the original version of this article contained errors, for which the publisher sincerely apologizes. The corrected details are given here.

The first paragraph which is missing under the heading "Case report" should be as below:

A 68-year-old woman, complaining of dysphagia, visited our hospital in 2019 and was diagnosed with a benign esophageal stricture in the lower esophagus due to severe GERD (Fig. 1a). She received vonoprazan (10 mg daily) as her first antacid treatment, alongside three sessions of endoscopic balloon dilatation for the stricture. Thereafter, we followed up regularly with the patient by endoscopy investigation. Her GERD symptoms did not flare up (Fig. 1b), but she developed multiple WGAs in her noncancerous stomach 1 year after starting vonoprazan. Further details of the case were described in our previous report [8].

The original article can be found online at https://doi.org/10.1007/ s12328-021-01437-z.

Wataru Miwa

wmiwa@ichou.gr.jp

1 Division of Internal Medicine, Hiratsuka Gastroenterological Hospital, 3-2-16 Nishiikebukuro Toshima-ku, Tokyo 171-0021, Japan

2 Division of Surgery, Hiratsuka Gastroenterological Hospital, Tokyo, Japan

3 Department of Cancer Genomic Medicine, Saitama Medical University International Medical Center, Saitama, Japan

4 Division of Pathology, Hiratsuka Gastroenterological Hospital, Tokyo, Japan
The reference citation should be changed from " $[2-8]$ " to "[8]" in the third sentence of the second paragraph in page 2 .

The reference citation should be changed from "[4-8]" to " $[4-6,8]$ " in the first sentence of the paragraph under the heading "Discussion".

In page 3, fourth paragraph under the heading "Case report", in the sixth sentence, the reference citation should be changed from " $[5,8]$ " to " $[8]$ ".

In figure 5 caption, sixth and seventh sentences should be as "c Specimen obtained from the vicinity of the WGA in Fig. 5a reveals marked PCP with oxyntic gland dilatation. d Specimen obtained from the vicinity of the WGA in Fig. 5b reveals that the glands are less tightly packed with slightly loosened and edematous stroma."

Publisher's Note Springer Nature remains neutral with regard to jurisdictional claims in published maps and institutional affiliations. 